\title{
Rapamycin reduces mortality in acute-stage paraquat-induced toxicity in zebrafish
}

\author{
Nan Feng ${ }^{1, *}$, MD, PhD, Zhaolian Bian ${ }^{2, *}$, MD, PhD, Xiaobin Zhang ${ }^{1}$, MD, Changsheng Wang ${ }^{1}$, MD, Jie $\underline{\text { Chen }}^{1}$, MD
}

INTRODUCTION Paraquat (PQ) intoxication is frequently associated with a high mortality rate. No specific treatment has been shown to reduce mortality in victims within the first 72 hours. We investigated the protective effects of rapamycin (Rapa) against PQ-induced toxicity in a zebrafish model.

METHODS To determine the maximum nonlethal concentration (MNLC) and lethal concentration 50 (LC50) of Rapa, zebrafish were treated at 2-5 days post fertilisation (dpf) and their mortality was recorded every 24 hours. At $5 \mathrm{dpf}$, the

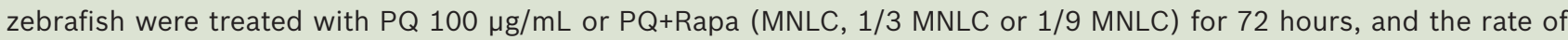
survival was recorded every 24 hours. Reverse transcription-polymerase chain reaction was used to test the signalling pathway of mTOR (mammalian target of rapamycin).

RESULTS MNLC and LC50 of Rapa were determined to be $6.7 \mu \mathrm{g} / \mathrm{mL}$ and $28.9 \mu \mathrm{g} / \mathrm{mL}$, respectively. At 48 hours, the $P Q+$ Rapa groups had much lower mortality than the $P Q$ group. The rates of survival of the $P Q+$ Rapa groups were $43.33 \%$ (MNLC), $53.89 \%$ (1/3 MNLC) and $44.45 \%$ (1/9 MLNC), as compared to $19.45 \%$ in the PQ group, with the $1 / 3 \mathrm{MNLC}$ group showing the highest rate of survival $(p<0.001)$. atg1 was slightly activated in the $P Q$ group. In the $P Q+R a p a$ groups, the expression of atg1 was markedly increased, suggesting strengthening of the autophagy process.

CONCLUSION Rapa can increase the rate of survival of PQ-intoxicated zebrafish by inhibiting mTOR complex 1 and activating autophagy. Rapa could be an alternative first-line drug in the treatment of $P Q$ poisoning.

Keywords: paraquat-induced toxicity, rapamycin, zebrafish

\section{INTRODUCTION}

Paraquat ( $\mathrm{PQ}, 1$ 1,1'-dimethyl-4,4'-bipyridinium dichloride), the most important member of a group of bipyridyl herbicides, has been widely used as a weedkiller since 1962. In humans, ingestion of $\mathrm{PQ}$, whether accidental or intentional, is frequently fatal and associated with a high rate of mortality. ${ }^{(1)}$ In general, an amount greater than $20 \mathrm{mg}$ of PQ ion per $\mathrm{kg}$ of body weight results in death. Shortly after the ingestion of large amounts of PQ (> $40 \mathrm{mg}$ ion per kg of body weight), multiple organ failure and cardiogenic shock rapidly occur, usually resulting in death within a few hours to a few days. When smaller amounts are ingested (20-40 mg), oxidative damage to the lungs, progressive pulmonary fibrosis and respiratory failure typically occur. ${ }^{(2,3)}$ To date, there is a lack of evidence supporting the effectiveness of therapeutic measures such as prevention of absorption, increasing excretion, and the use of immunosuppressive and antioxidant treatments. A randomised controlled trial has shown that treatment with repeated pulses of methylprednisolone and cyclophosphamide with continuous dexamethasone is promising. ${ }^{(4)}$ However, a systematic search for human studies by Gawarammana and Buckley demonstrated that the fatality rates of PQ poisoning remained very high in all centres despite the diverse treatments. ${ }^{(5)}$

PQ can induce apoptosis by producing reactive oxygen species and activating nuclear factor-kappa $\mathrm{B}$. This results in nuclear condensation and DNA fragmentation. ${ }^{(5)}$ Thus, PQ is frequently used for modelling Parkinson's disease. ${ }^{(6,7)} \mathrm{PQ}$ has also been proven to increase autophagy in fibroblasts. ${ }^{(8)}$

Rapamycin (Rapa) is a macrocyclic triene antibiotic produced by the soil bacterium Streptomyces hygroscopicus. It is widely used as an immunosuppressive agent for patients after solid-organ transplantation. Ravikumar et al reported that wild-type flies that were pretreated with Rapa, an mTOR (mammalian target of rapamycin) inhibitor, showed an increased rate of survival after exposure to $\mathrm{PQ}$ and that this protective effect is accounted for largely or entirely by autophagy. ${ }^{(9)}$ In a study involving male Lewis rats, the application of Rapa was shown to halt the development of pulmonary fibrosis induced by bleomycin. ${ }^{(10)}$ A few other studies have shown the positive effect of Rapa on lung injury in rats, ${ }^{(11,12)}$ although a case study reported that Rapa failed to stop pulmonary fibrosis from progressing in a patient with $\mathrm{PQ}$ intoxication. ${ }^{(13)}$

In Ren Ji Hospital, Shanghai Jiao Tong University School of Medicine, Shanghai, China, cases of PQ intoxication are treated with comprehensive therapies comprising high-dose methylprednisolone, cyclophosphamide and haemoperfusion. However, most of these patients die due to multiple organ system failure within 72 hours of ingesting high-dose PQ. The majority of published studies on PQ intoxication have focused on lung injury, lower PQ dosages and longer duration of survival in animal models. The present study aimed to investigate whether Rapa decreases mortality in the first three days of PQ ingestion. If Rapa proves to be effective, clinicians may have more time to try out

\footnotetext{
${ }^{1}$ Emergency Department, Ren Ji Hospital, School of Medicine, Shanghai Jiao Tong University, Shanghai, ${ }^{2}$ Department of Gastroenterology and Hepatology, Nantong Institute of Liver Disease, Nantong Third People's Hospital, Nantong, Jiangsu, China

*These two authors contributed equally as first author in this work.
}

Correspondence: Dr Jie Chen, Associate Chief Physician, Emergency Department, Ren Ji Hospital, School of Medicine, Shanghai Jiao Tong University, Shanghai 200127, People's Republic of China. cjrenji@126.com 
various treatments, thereby increasing the chances of survival. In this study, we evaluated the protective effect of Rapa against fatal PQ intoxication in a zebrafish model.

\section{METHODS}

Parental-generation adult wild-type $A B$ strain zebrafish were raised and maintained under standard conditions. Zebrafish roe was incubated in separate Petri dishes. The Animal Care and Use Committee of the Shanghai Research Center for Model Organisms, Shanghai, China, approved the animal procedures described in this article. The procedures were consistent with the guidelines set out by the American Veterinary Medical Association's Panel on Euthanasia.

To determine the maximum nonlethal concentration (MNLC) and lethal concentration 50 (LC50) of Rapa (LC Laboratories, Woburn, MA, USA), zebrafish were treated at 2-5 days post fertilisation (dpf) and mortality was recorded every 24 hours. Death was defined as the absence of a heartbeat under a dissecting stereomicroscope (SMZ645; Nikon, Tokyo, Japan). Drug intervention was performed in 12-well plates (BD Falcon; BD Biosciences, San Jose, CA, USA) with 30 zebrafish in each well. Triplicate wells were used for each treatment, and the experiment was repeated at least twice independently. Mortality curves were generated using GraphPad Prism 5.0 (GraphPad Software, San Diego, CA, USA) and the MNLC was determined using logistic regression.

Initial tests were conducted to determine the MNLC and LC50 of Rapa (Sigma, St Louis, MO, USA). One control group received $0.1 \%$ dimethyl sulfoxide (DMSO) and five other groups received Rapa in concentrations of $0.1 \mu \mathrm{g} / \mathrm{mL}, 1 \mu \mathrm{g} / \mathrm{mL}$, $10 \mu \mathrm{g} / \mathrm{mL}, 100 \mu \mathrm{g} / \mathrm{mL}$ and 1,000 $\mu \mathrm{g} / \mathrm{mL}$. Subsequently, we tested 11 concentrations of Rapa $(0.1 \mu \mathrm{g} / \mathrm{mL}, 0.5 \mu \mathrm{g} / \mathrm{mL}, 1 \mu \mathrm{g} / \mathrm{mL}$, $5 \mu \mathrm{g} / \mathrm{mL}, 10 \mu \mathrm{g} / \mathrm{mL}, 25 \mu \mathrm{g} / \mathrm{mL}, 50 \mu \mathrm{g} / \mathrm{mL}, 100 \mu \mathrm{g} / \mathrm{mL}, 250 \mu \mathrm{g} / \mathrm{mL}$, $500 \mu \mathrm{g} / \mathrm{mL}$ and $1,000 \mu \mathrm{g} / \mathrm{mL}$ ), with the control group receiving the same concentration of DMSO (i.e. $0.1 \%$ ). When the concentration of Rapa exceeded $1,000 \mu \mathrm{g} / \mathrm{mL}$, the crystals separated out into the water.

There was no mortality in the control group and the first five groups (i.e. Rapa $0.1 \mu \mathrm{g} / \mathrm{mL}, 0.5 \mu \mathrm{g} / \mathrm{mL}, 1 \mu \mathrm{g} / \mathrm{mL}, 5 \mu \mathrm{g} / \mathrm{mL}$, $10 \mu \mathrm{g} / \mathrm{mL}$ and $25 \mu \mathrm{g} / \mathrm{mL}$ ). Mortality rates rose with a corresponding increase in the concentration of Rapa (Appendix; Supplementary Fig. 1a). Four-parameter logistic equation was used to estimate the MNLC and LC50 of Rapa (Appendix; Supplementary Fig. 1b), which were determined to be $6.7 \mu \mathrm{g} / \mathrm{mL}$ and $28.9 \mu \mathrm{g} / \mathrm{mL}$, respectively.

To assess the protective effects of Rapa against PQ-induced toxicity, five-day-old zebrafish were given either PQ $100 \mu \mathrm{g} / \mathrm{mL}$ or PQ $100 \mu \mathrm{g} / \mathrm{mL}$ plus Rapa (MNLC: $6.7 \mu \mathrm{g} / \mathrm{mL}$; 1/3 MNLC: $2.2 \mu \mathrm{g} / \mathrm{mL}$; or $1 / 9 \mathrm{MNLC}$ : $0.8 \mu \mathrm{g} / \mathrm{mL}$ ) for 72 hours. The rate of survival was recorded every 24 hours. Based on the above, the zebrafish were divided into four groups: $\mathrm{PQ} ; \mathrm{PQ}+$ Rapa (MNLC); PQ+Rapa (1/3 MNLC); and PQ+Rapa (1/9 MNLC). The control group received $0.1 \%$ DMSO.

Embryos and larvae were analysed using a fluorescence microscope (SMZ1500; Nikon, Tokyo, Japan) and subsequently photographed using a digital camera. A subset of images was adjusted for levels, brightness, contrast, hue and saturation using Adobe Photoshop 7.0 (Adobe, San Jose, CA, USA) to optimally visualise the patterns of expression. Quantitative image analyses were processed using ImageJ software (National Institutes of Health, Bethesda, MD, USA). Inverted fluorescence images were used for processing. Positive signals were defined by particle number using ImageJ. Ten animals were quantified for each treatment and the total signal per animal was averaged.

Zebrafish samples (3 dpf) were processed for extraction of RNA using TRIzol reagent (Invitrogen; Thermo Fisher Scientific, Waltham, MA, USA), and complementary DNA was synthesised according to the manufacturer's instructions (Fermentas, Burlington, ON, Canada). Reverse transcription-polymerase chain reaction (RT-PCR) was performed with a green-based PCR approach using SYBR Premix Ex Taq ${ }^{\text {TM }}$ (Takara, Shiga, Japan) and Roche LightCycler ${ }^{\circledR} 480$ (Roche, Indianapolis, IN, USA). Gene expression was normalised to actb1. All primer sequences are presented in Supplementary Table I (Appendix).

All data was presented as mean \pm standard error of mean. Statistical analysis and graphical representation of data were performed using GraphPad Prism 5.0 (GraphPad Software). For comparisons between two groups, two-tailed $t$-test assuming unequal variances was used. For comparisons between more than two groups, one-way analysis of variance (ANOVA), followed by Bonferroni multiple comparisons, was used to compare each data point with the control. For experiments with multiple groups, two-way ANOVA tests followed by Bonferroni post-hoc tests were used. Statistical significance was set at $p<0.05$ and $p<0.001$.

\section{RESULTS}

On Day 1 (24 hours), no significant difference in the rate of survival $(p>0.5)$ was observed in the PQ+Rapa groups as compared to the PQ group. On Day 2 (48 hours), the mean rates of survival in the PQ+Rapa groups were MNLC: $43.33 \%$; 1/3 MNLC: 53.89\%; and $1 / 9$ MNLC: $44.45 \%$ (Table I \& Fig. 1 ). The mortality rate was much lower in the $\mathrm{PQ}+$ Rapa groups compared to the $\mathrm{PQ}$ group, with the $1 / 3$ MNLC group showing the highest rate of survival as compared to the PQ group ( $p<0.001)$. On Day 3 (72 hours), all the zebrafish in the PQ group had died, while a few zebrafish in the PQ+Rapa groups were still alive. The mean survival rates in the PQ+Rapa groups were MNLC: $3.89 \% ; 1 / 3$ MNLC: $7.78 \%$; and 1/9 MNLC: $3.89 \%$.

We tested the expression of $m T O R$ in the control, PQ and PQ+Rapa (1/3 MNLC) groups. Results of quantitative PCR showed that $m T O R$ was significantly inhibited in the $P Q+$ Rapa (1/3 MNLC) group as compared to the control group, leading to a mild increase in the expression of atg1 (ulk1a, ulk1b and ulk3), which may be the key factors in autophagy. We also found that when treated with Rapa, the expression of $m T O R$ was upregulated, as seen in the PQ+Rapa (1/3 MNLC) group compared to the PQ group $(p<0.0001)$, and atg1 was significantly activated. atg13, another important factor in the process of autophagy, was downregulated in the PQ group and upregulated in the PQ+Rapa (1/3 MNLC) group (Fig. 2). 
Table I. Survival rates of the groups of zebrafish.

\begin{tabular}{|lllll|}
\hline Treatment group & \multicolumn{4}{c|}{ Mean \pm standard error of mean (\%) } \\
\cline { 2 - 5 } & Day 0 & Day 1 & Day 2 & Day 3 \\
\hline Control & $100.00 \pm 0$ & $100.00 \pm 0$ & $100.00 \pm 0$ & $100.00 \pm 0$ \\
\hline PQ only & $100.00 \pm 0$ & $92.22 \pm 1.859$ & $19.45 \pm 2.777$ & $0 \pm 0$ \\
\hline PQ+Rapa (MNLC) & $100.00 \pm 0$ & $89.44 \pm 2.908$ & $43.33 \pm 5.643$ & $3.89 \pm 2.180$ \\
\hline PQ+Rapa (1/3 MNLC)* & $100.00 \pm 0$ & $94.45 \pm 1.859$ & $53.89 \pm 2.003$ & $7.78 \pm 2.533$ \\
\hline PQ+Rapa (1/9 MNLC) & $100.00 \pm 0$ & $92.22 \pm 3.913$ & $44.45 \pm 5.879$ & $3.89 \pm 2.003$ \\
\hline
\end{tabular}

*Statistically significant at $p<0.001$ compared with the PQ group. MNLC: maximum nonlethal concentration; PQ: paraquat; Rapa: rapamycin

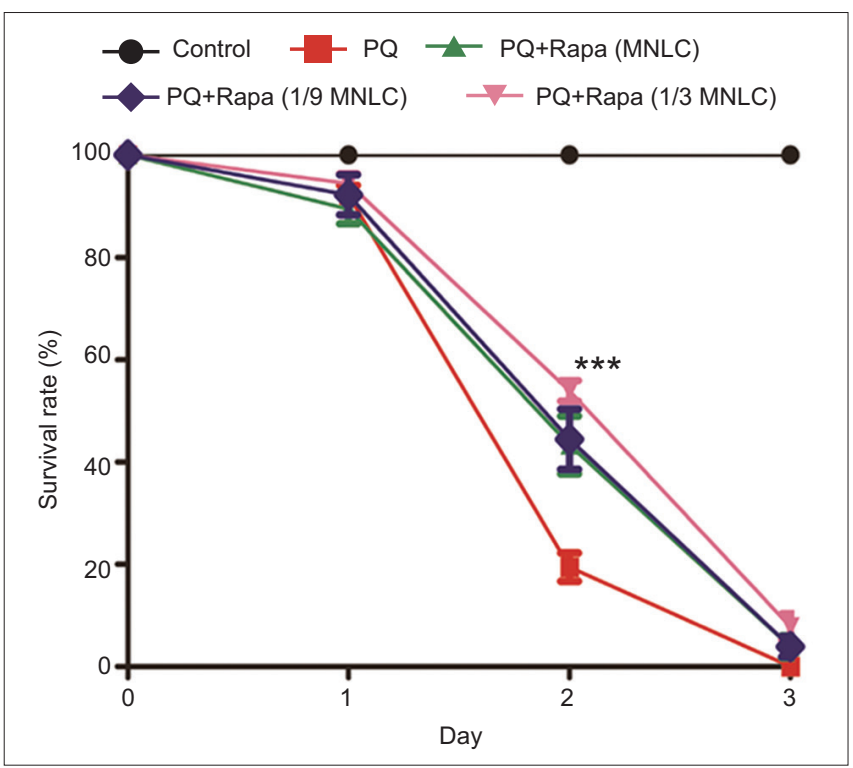

Fig. 1 Graph shows the survival rates of different treatment groups of zebrafish. $p$-value is statistically significant at *** $p<0.001(P Q+R a p a$ group vs. $P Q$ group). MNLC: maximum nonlethal concentration; $P Q$ : paraquat; Rapa: rapamycin

We also studied the roles of different mTOR pathways in the PQ-induced model, as mTOR senses hypoxia, energy stress, amino acid levels and insulin levels. Cells encountering hypoxic stress conserve resources and energy by downregulating the synthesis of protein. It has been reported that the knockout of tuberous sclerosis complex 1 or 2 (Tsc1/Tsc2) or the overexpression of Ras homologue enriched in brain (rheb) rescues terminal oligopyrimidine mRNA translation in oxygen-deprived cells. ${ }^{(14)}$ Redd1 (regulated in development and DNA damage response 1), also known as ddit4 (DNA-damage-inducible transcript 4), is an inhibitor of mTOR, which senses hypoxia by regulating Tsc. ${ }^{(15)}$ In the PQ group (vs. the control group), the level of expression of ddit4 was slightly decreased; tsc $1 a$, tsc $1 b$ and tsc 2 expressions were upregulated, while the expression of rheb was significantly upregulated, leading to the downregulation of $m T O R$. In the $\mathrm{PQ}+$ Rapa (1/3 MNLC) group, the level of ddit4 was high, and the level of expression of tsc was markedly higher than that in the PQ group; the rheb level was high as compared to the control group but lower as compared to the PQ group (Fig. 3a). The PQ+Rapa (1/3 MNLC) group had significantly higher expression of $m T O R$ than both the control and PQ groups.

Another pathway, activation of mTOR complex 1 (mTORC1) by amino acids, is mediated in part by Rag GTPases, which bind
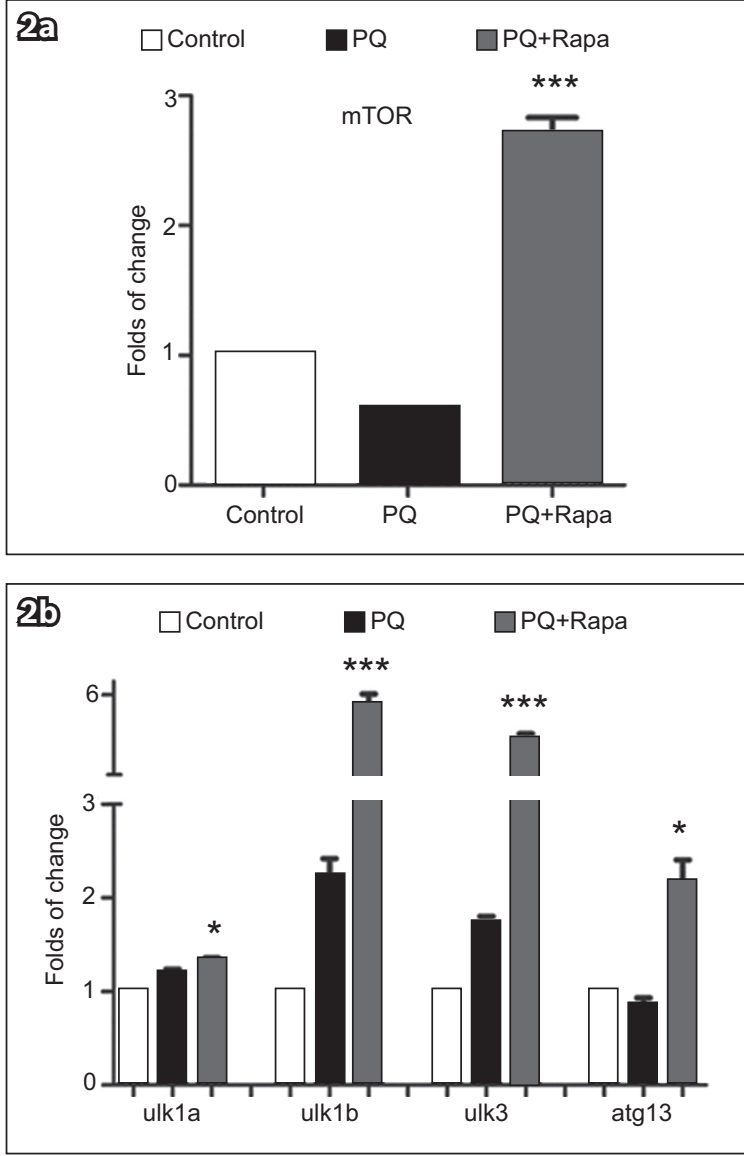

Fig. 2 Expression of mTOR and atgs. Graph shows (a) significantly upregulated expression of mTOR in the $P Q+$ Rapa (1/3 MNLC) group ( $p<0.001$ ); and (b) upregulated ulk1a, ulk1b, ulk3 and atg13 in the $P Q+$ Rapa (1/3 MNLC) group vs. the $P Q$ group. $p$-value is statistically significant at ${ }^{*} p<0.05$ and ${ }^{* * *} p<0.001$. mTOR: mammalian target of rapamycin; PQ: paraquat; Rapa: rapamycin

the raptor subunit of mTORC1 in an amino acid-stimulated manner and promote the interaction of mTORC1 with Rheb-GTP, the immediate activator. ${ }^{(16)}$ In the PQ group, the expression of rragd (Ras-related GTP-binding D), rragca (Ras-related GTPbinding $\mathrm{Ca}$ ) and rraga (Ras-related GTP-binding A) was slightly downregulated; the expression of rragcb (Ras-related GTP-binding $\mathrm{Cb}$ ) was almost at the same level as in the control group. In the PQ+Rapa (1/3 MNLC) group, the expression of rragd, rragca and rragcb was elevated as compared to the PQ group ( $p<0.05)$, but no difference in rraga was found between the two groups (Fig. 3b).

Phosphoinositide 3-kinase (PI3K)-protein kinase B (AKT), an important signalling pathway that regulates mTOR, is affected by growth factor and insulin level. Phosphatase and tensin 


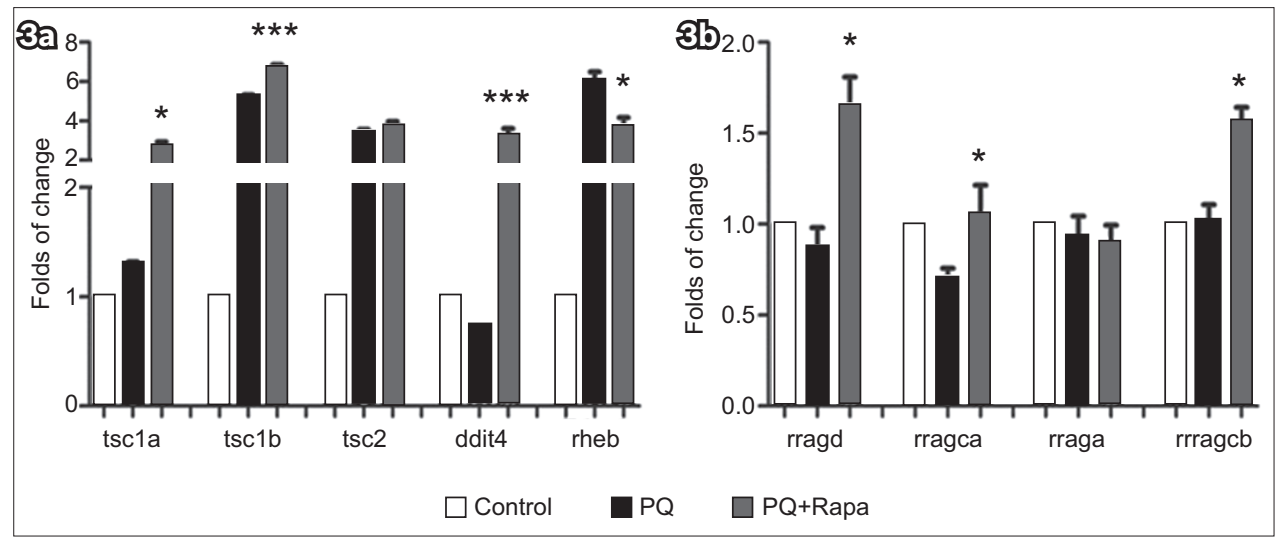

Fig. 3 Expression of tsc, ddit4, rheb and rrags. (a) Graph shows upregulated tsc1a and tsc1b in the PQ+Rapa (1/3 MNLC) group vs. the $P Q$ group. Although the tsc2 level was high in both the $P Q$ and $P Q+$ Rapa (1/3 MNLC) groups, there was no significant difference between the two groups $(p=0.15)$. rheb was negatively related to ddit4. (b) Graph shows different expression of rrags after intervention in the PQ+Rapa $(1 / 3$ MNLC) group $(p=0.79)$. $p$-value is statistically significant at ${ }^{*} \mathrm{p}<0.05$ and ${ }^{* * *} \mathrm{p}<0.001$. ddit4: DNA-damage-inducible transcript 4; MNLC: maximum nonlethal concentration; $\mathrm{PQ:} \mathrm{paraquat;}$ Rapa: rapamycin; rheb: Ras homologue enriched in brain; rrags: Ras-related GTP-binding protein; tsc: tuberous sclerosis complex

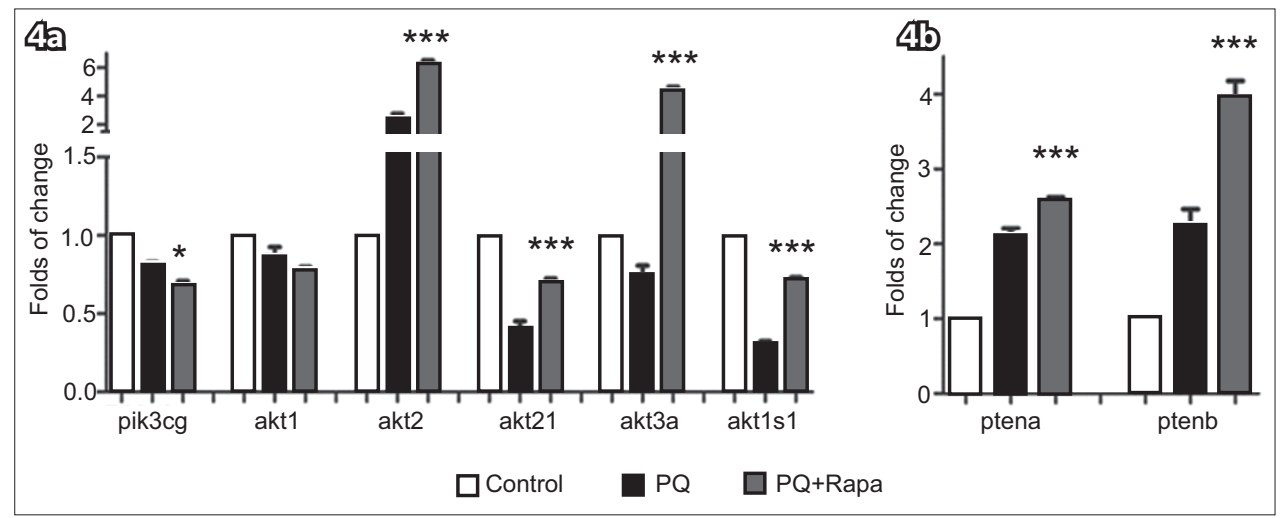

Fig. 4 Expression of PI3K pathway. (a) Graph shows inhibited pik3cg in both the $P Q$ and $P Q+$ Rapa ( $1 / 3$ MNLC) groups. Its downstream factors, the akts, were also influenced by pik3cg and mTORC1. In the PQ+Rapa (1/3 MNLC) group, akt1 was lower as compared to the PQ group, but this was not statistically significant ( $p=0.0706)$; (b) Graph shows abnormally activated ptena and ptenb in both the intervention groups, although they were higher in the $P Q+R a p a(1 / 3 \mathrm{MNLC})$ group. $p$-value is statistically significant at ${ }^{*} p<0.05$ and ${ }^{* * *} p<0.001$. akt: protein kinase $B$; PI3K: phosphoinositide 3-kinase; pik3cg: PI3K catalytic subunit gamma; PQ: paraquat; ptena and ptenb; phosphatase and tensin homologue A and B; Rapa: rapamycin

homologue A and B (PTENa and PTENb), which are lipid and protein phosphatases, antagonise the PI3K-AKT pathway by balancing the cellular phosphatidylinositol 3,4,5-trisphosphate level. pik3cg (phosphatidylinositol-4,5-bisphosphate 3-kinase, catalytic subunit gamma) was inhibited in both the PQ group and PQ+Rapa (1/3 MNLC) group (Fig. 4). ptena and ptenb were upregulated and significantly greater $(p<0.001)$ in the $P Q+$ Rapa (1/3 MNLC) group (Fig. 4b). akt (akt1, akt21 and akt3a) other than akt2 were downregulated in the PQ group as compared to the control group. In the PQ+Rapa (1/3 MNLC) group, expression of all decreasing akts was recovered; akt3a was higher than that in the control group and akt2 was even higher than that in the PQ group (Fig. 4a). In addition, akt1s1 (also known as PRAS40) was significantly downregulated in the PQ group but partially recovered when the zebrafish were treated with Rapa (Fig. 4a).

\section{DISCUSSION}

In our centre, more than 20 patients are admitted due to PQ poisoning every year. A majority of them are young people who commit or threaten suicide. PQ is often chosen as the poison of choice (instead of organophosphorus or other pesticides), as it is often misleadingly labelled on the PQ bottle as 'hypotoxic'. This could be a marketing tactic aimed to boost sales of the product and is a serious public health problem that the government should address.

Many studies have pointed out that medical treatment with glucocorticoid, cytoxan or bosentan might be effective in the treatment of PQ poisoning. ${ }^{(17,18)}$ However, most of these are case reports. Two of our previous studies have shown that FTY20 and deoxyribonuclease I might be promising approaches for the treatment of PQ-induced acute lung injury and pulmonary fibrosis. ${ }^{(19,20)}$ Some studies have evaluated the therapeutic role of Rapa against PQ poisoning in rat and mice models, ${ }^{(11,12)}$ but they have mostly focused on chronic poisoning. In this study, we verified the protective effect of Rapa against high-dose PQ poisoning using a zebrafish model, because the zebrafish has been found to be more sensitive to toxicological evaluation than other animals. ${ }^{(21)}$

Our results showed that the rate of mortality was high in PQintoxicated zebrafish. However, the rate of survival at 48 hours was $23.88 \%-34.44 \%$ higher in zebrafish treated with Rapa than those not treated with Rapa. Furthermore, at the end of the study (72 hours), the PQ+Rapa (1/3 MNLC) group showed the highest rate of survival as compared to the PQ group $(7.78 \% ; p=0.012)$.

We subsequently tested the mTOR signalling pathway using RT-PCR. As Ddit4 senses hypoxia, its activation would indirectly 
upregulate Tsc. PQ-induced intoxication revealed the following: the Ddit4/Tsc/Rheb pathway and mTOR were inhibited; the expression of rheb was abnormally high; and ulk (atg1) and the autophagy process were slightly activated. After treatment with Rapa, the expression of mTOR was increased, but mTORC1 was theoretically inhibited, which resulted in marked upregulation of $u l k 1 a, u l k 1 b$ and $u l k 3$. Rapa resulted in upregulation of the expression of ddit4, as well as tsc $1 a$, tsc $1 b$ and tsc2; the expression of rheb was lower than in the group treated with PQ only.

mTOR is also activated through Rag GTPases/mTORC1. In the present study, PQ induced a slightly lower expression of rag and inhibition of $m T O R$. In the PQ+Rapa (1/3 MNLC) group, the expression of Rag GTPases (rragd, rragca, rraga and rragcb) increased, which resulted in increased expression of $m T O R$. In addition, a change in the expression of the PI3K/AKT/PRAS40 pathway was also observed: pik3cg was inhibited and ptens was activated; the former regulates akt by phosphorylation and the latter blocks the phosphorylation of PI3K, all of which led to a change in the expression of akts. Most akts, with the exception of akt2, were inhibited. Hence, akt2 appeared to block akt1s1, which downregulated mTORC1 directly.

Therefore, we opine that PQ could inhibit the expression of mTOR via several pathways and moderately activate the autophagy pathway. We also postulate that $\mathrm{PQ}+$ Rapa could affect the upstream pathway in regulating the expression of mTOR, although this mechanism needs to be further studied.

The 72-hour mortality rate in our study was very high in all groups, which was expected. However, our higher survival rate at 48 hours was noteworthy, and a few points could be extrapolated from this. Firstly, only Rapa was used in our study; this could have resulted in the reduced mortality rate during the acute period and provided more time for comprehensive treatments to be effective. Secondly, at 72 hours, we observed that a few zebrafish in the PQ+Rapa (1/3 MNLC) group were still alive and only minor differences were noted in the 72 hour mortality rates among the groups receiving different doses of Rapa. Therefore, we speculate that a low dose of Rapa is potentially sufficient to serve as an effective first-line therapy for PQ poisoning. Contrary to our observation, Lorenzen et al reported a case of failed rescue therapy with Rapa $8 \mathrm{mg} / \mathrm{kg}$ (and high-dose methylprednisolone $1 \mathrm{~g}$ /day), which was started three days after PQ intoxication. ${ }^{(13)}$ Thirdly, Rapa could increase the rate of survival of zebrafish poisoned by PQ by inhibiting mTORC1.

In conclusion, based on the results of the present study, we recommend that all PQ poisoning treatments that have purported effects, such as high-dose prednisolone or dexamethasone, cyclophosphamide, salicylate, haemoperfusion, acetylcysteine and salicylate, should be started as soon as possible. Rapa $(8 \mathrm{mg} / \mathrm{kg})$ is a potentially effective first-line therapy and should be administered concurrently with other treatments after informed consent is obtained.

\section{REFERENCES}

1. Sabzghabaee AM, Eizadi-Mood N, Montazeri K, Yaraghi A, Golabi M. Fatality in paraquat poisoning. Singapore Med J 2010; 51:496-500.

2. Agarwal R, Srinivas R, Aggarwal AN, Gupta D. Immunosuppressive therapy in lung injury due to paraquat poisoning: a meta-analysis. Singapore Med J 2007; 48:1000-5.

3. Newstead CG. Cyclophosphamide treatment of paraquat poisoning. Thorax 1996; 51:659-60.

4. Lin JL, Lin-Tan DT, Chen KH, Huang WH. Repeated pulse of methylprednisolone and cyclophosphamide with continuous dexamethasone therapy for patients with severe paraquat poisoning. Crit Care Med 2006; 34:368-73.

5. Gawarammana IB, Buckley NA. Medical management of paraquat ingestion. Br J Clin Pharmacol 2011; 72:745-57.

6. Corasaniti MT, Strongoli MC, Rotiroti D, Bagetta G, Nisticò G. Paraquat: a useful tool for the in vivo study of mechanisms of neuronal cell death. Pharmacol Toxicol 1998; 83:1-7.

7. Bortolotto JW, Cognato GP, Christoff RR, et al. Long-term exposure to paraquat alters behavioral parameters and dopamine levels in adult zebrafish (Danio rerio). Zebrafish 2014; 11:142-53.

8. Wang M, Miller RA. Fibroblasts from long-lived mutant mice exhibit increased autophagy and lower TOR activity after nutrient deprivation or oxidative stress. Aging Cell 2012; 11:668-74.

9. Ravikumar B, Berger Z, Vacher C, O'Kane CJ, Rubinsztein DC. Rapamycin pre-treatment protects against apoptosis. Hum Mol Genet 2006; 15:1209-16.

10. Simler NR, Howell DC, Marshall RP, et al. The rapamycin analogue SDZ RAD attenuates bleomycin-induced pulmonary fibrosis in rats. Eur Respir J 2002; 19:1124-7.

11. Shao X, Li M, Luo $C$, et al. Effects of rapamycin against paraquat-induced pulmonary fibrosis in mice. J Zhejiang Univ Sci B 2015; 16:52-61.

12. Chen D, Jiao G, Ma T, et al. The mechanism of rapamycin in the intervention of paraquat-induced acute lung injury in rats. Xenobiotica 2015; 45:538-46.

13. Lorenzen JM, Schonenberger E, Hafer C, Hoeper M, Kielstein JT. Failed rescue therapy with rapamycin after paraquat intoxication. Clin Toxicol (Phila) 2010; 48:84-6.

14. Miloslavski R, Cohen E, Avraham A, et al. Oxygen sufficiency controls TOP mRNA translation via the TSC-Rheb-mTOR pathway in a 4E-BP-independent manner. J Mol Cell Biol 2014; 6:255-66.

15. Han J, Xiao Z, Chen L, et al. Maintenance of the self-renewal properties of neural progenitor cells cultured in three-dimensional collagen scaffolds by the REDD1-mTOR signal pathway. Biomaterials 2013; 34:1921-8.

16. Oshiro N, Rapley J, Avruch J. Amino acids activate mammalian target of rapamycin (mTOR) complex 1 without changing Rag GTPase guanyl nucleotide charging. J Biol Chem 2014; 289:2658-74.

17. Zhang Q, Wu WZ, Lu YQ, et al. Successful treatment of patients with paraquat intoxication: three case reports and review of the literature. J Zhejiang Univ Sci B 2012; 13:413-8.

18. Zhang Z, Jian X, Zhang W, Wang J, Zhou Q. Using bosentan to treat paraquat poisoning-induced acute lung injury in rats. PLoS One 2013; 8:e75943.

19. Qian J, Ye Y, Lv L, Zhu C, Ye S. FTY720 attenuates paraquat-induced lung injury in mice. Int Immunopharmacol 2014; 21:426-31.

20. Li G, Yuzhen L, Yi C, et al. DNasel protects against paraquat-induced acute lung injury and pulmonary fibrosis mediated by mitochondrial DNA. Biomed Res Int 2015; 2015:386952.

21. Zon LI, Peterson RT. In vivo drug discovery in the zebrafish. Nat Rev Drug Discov 2005; 4:35-44. 


\section{APPENDIX}
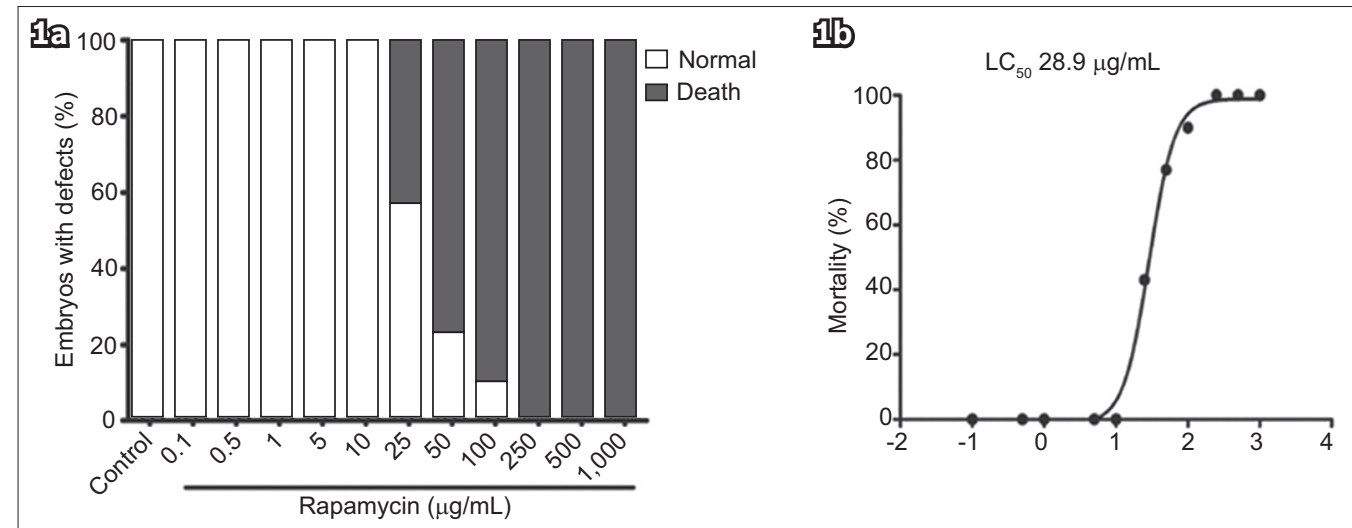

Supplementary Fig. 1 Quantitative analysis of the toxicity and safety of rapamycin in the zebrafish model. Data consists of three-day post-fertilisation statistics. Graph shows (a) the percentage of embryos with defects after rapamycin treatment; and (b) the maximum nonlethal concentration of rapamycin. LC50: lethal concentration 50

Supplementary Table I. Primer sequences.

\begin{tabular}{|c|c|c|}
\hline & Forward & Reverse \\
\hline mTOR & CGATGAACTGAACCACCACA & ТССТСССТССАСТССААТС \\
\hline ulk1a & TACGCACACACCGTTCAGAT & GCCCTCCATCAGCAGTAAGG \\
\hline ulk1b & GAAGGAATCAAGCAGGGAAA & CAGAGACCGACAGGACATCA \\
\hline ulk3 & GTCCGTCGGCGTCATTCTAT & GTAGATCCCTGCAGTCACGG \\
\hline $\operatorname{atg} 13$ & AGCCCACAGGACAAGAAAGA & GGTGATGAAGACGAGCAAGTG \\
\hline tsc1a & CTGTCGTCTCTCCACCATCA & TGAGGCATTTGAGCAGTGAG \\
\hline tsc1b & GGCAGAGGGTGTTACTGAGC & GGAGACTGATGTGGCTTTCC \\
\hline tsc2 & GATTGAGGTGGTGGTCATCC & CTGCTGAAGTGGTGTGTGCT \\
\hline ddit4 & CGCCTTTGTTCGCTCTTAGT & GTGTGGACGGACTGGAATCT \\
\hline rheb & TCCTCCCTTACGATACAGTTTG & CTTGACCATTCACAGACACCA \\
\hline rregd & CCGTTCAGCAGTGAGGTGA & TGGGTGACATCTTGTGGAAA \\
\hline rragca & CTCCCTCCGACAGTAAACCA & GTCTCATTGGGCGACATCTT \\
\hline rraga & GGACTGTQCTGGTCAGGATA & TCTCTAACTCTCGGCTCTCCA \\
\hline rragcb & TGCGAAGTCCACACAGACAT & AAGAGTCCACCAGGCTACCG \\
\hline pik3cg & AACAACTGCTGGCTCAAGAAA & GGCACCTTTGGGTAAGTCCT \\
\hline akt1 & GAGAACCTGATGCTGGATA & ATAGTGGCTCCGTCTGTGA \\
\hline akt2 & CGGTTTGTGAGGAGAATCGT & CGAGGGTGTTTAGAGGCTTG \\
\hline akt21 & TCTGCACTCACAGAACGTGG & GCACGGCCGTAATCATTGTC \\
\hline akt3a & ACGACCAAAGCCGAACACT & ССАСТСАТСССТСТССТСТG \\
\hline akt1s1 & GCT CCG AAT AAA GAG GGC G & TGG GTG ATG GTG TAT GCG T \\
\hline ptena & AGCCCTCAAACTCAGAAGCA & CGGGTCAGAATCAGTGGTGT \\
\hline ptenb & ATTCCCAGTCAGAGGCGATA & AGGCACAGTCTCAAACACCA \\
\hline actb1 & CAT CGT TCA CAG GAA GTG C & GAG TTT AGG TTG GTC GTT C \\
\hline
\end{tabular}

